\title{
Lack of association between the Serotonin Transporter Promoter Polymorphism (5-HTTLPR) and Panic Disorder: a systematic review and meta-analysis
}

\author{
Carolina Blaya1, Giovanni A Salum¹, Maurício S Lima², Sandra Leistner- \\ Segal $^{1}$ and Gisele G Manfro*1
}

Address: ${ }^{1}$ Post-Graduate Program in Medical Sciences, Psychiatry, Universidade Federal do Rio Grande do Sul and Anxiety Disorders Program, Hospital de Clínicas de Porto Alegre, Porto Alegre, Brazil and ${ }^{2}$ Associate Professor of Psychiatry, Universidade Católica de Pelotas \& Medical Director, Eli Lilly do, Brazil

Email: Carolina Blaya - cblaya@terra.com.br; Giovanni A Salum - gsalumjr@gmail.com; Maurício S Lima - LIMA_MAURICIO_SILVA_DE@LILLY.COM; Sandra Leistner-Segal - ssegal@hcpa.ufrgs.br;

Gisele G Manfro* - gmanfro@portoweb.com.br

* Corresponding author

Published: 18 August 2007

Behavioral and Brain Functions 2007, 3:4I doi:|0.1|86/I744-908I-3-4I
Received: 6 July 2007

Accepted: 18 August 2007

This article is available from: http://www.behavioralandbrainfunctions.com/content/3/l/4 I

(c) 2007 Blaya et al; licensee BioMed Central Ltd.

This is an Open Access article distributed under the terms of the Creative Commons Attribution License (http://creativecommons.org/licenses/by/2.0), which permits unrestricted use, distribution, and reproduction in any medium, provided the original work is properly cited.

\begin{abstract}
Background: The aim of this study is to assess the association between the Serotonin Transporter Promoter Polymorphism (5-HTTLPR) and Panic Disorder (PD).

Methods: This is a systematic review and meta-analysis of case-control studies with unrelated individuals of any ethnic origin examining the role of the 5-HTTLPR in PD according to standard diagnostic criteria (DSM or ICD). Articles published in any language between January 1996 and April 2007 were eligible. The electronic databases searched included PubMed, Psychlnfo, Lilacs and ISI. Two separate analyses were performed: an analysis by alleles and a stratified analysis separating studies by the quality of control groups. Asymptotic DerSimonian and Laird's $Q$ test were used to assess heterogeneity. Results of individual studies were combined using the fixed effect model with respective $95 \%$ confidence intervals.
\end{abstract}

Results: Nineteen potential articles were identified, and 10 studies were included in this metaanalysis. No statistically significant association between $5-H T T L P R$ and PD was found, OR $=0.91$ $(\mathrm{Cl} 195 \% 0.80$ to $1.03, \mathrm{p}=0.14)$. Three sub-analyses divided by ethnicity, control group quality and Agoraphobia comorbidity also failed to find any significant association. No evidence of heterogeneity was found between studies in the analyses.

Conclusion: Results from this systematic review do not provide evidence to support an association between 5-HTTLPR and PD. However, more studies are needed in different ethnic populations in order to evaluate a possible minor effect.

\section{Background}

Studies in families have shown that Panic Disorder (PD) has a familiar pattern: its prevalence is higher in first degree family members than in control groups [1]. Studies involving siblings show that PD concordance is higher in monozygotic than dizygotic twins [2]. These findings pro- 
pose that genetic factors contribute to the pathogenesis of PD with an estimated heritability of 30-40\% [3], whereas a recent meta-analysis suggests a higher heritability of $48 \%[4]$.

Studies on candidate genes for association have been selected on the basis of the molecular therapeutic drugs and panic-provoking agents [5]. For instance, the response shown by panic patients when treated with Serotonin Selective Reuptake Inhibitors (SSRIs) and the worsening when using a serotoninergic agonist suggest a possible serotoninergic dysfunction in this disorder $[2,6]$.

The serotoninergic transporter gene (5-HTT) is located in chromosome 17q11.1-q12 [7] and it codes for a membrane protein that reuptakes serotonin from the synaptic cleft. A size repetition polymorphism has been related to functionality of the serotoninergic transporter protein. The polymorphism is a $44 \mathrm{bp}$ insertion or deletion on the promoter gene region ( 5 - HTTLPR) resulting in two alleles (l-long and s-short). The l allele transcription is two or three times more efficient than the s allele $[8,9]$. The $\mathrm{s}$ allele is less active, therefore, resulting in lower serotonin reuptake and, consequently, in increased serotonin in the synaptic cleft [10].

Previous studies found a significant association between $\mathrm{s}$ allele and anxiety traits in healthy volunteers [11]. Regarding PD, studies have systematically failed to find any association between this disorder and 5-HTTLPR. This lack of association could be related to the small sample size of studies. However, a recent study has raised the possibility that the $\mathrm{l}$ allele could be involved in panic disorder [12].

Although the controversies between 5-HTTLPR in PD may be related to methodological differences between studies, such as ethnicity, another limitation is lack of statistical power [13]. According to Hirschhorn et al. [14], out of 166 studies on gene-disease associations, only six replicated previous findings. Possible causes for this inconsistency include studies with small sample sizes $[15,16]$, as the most realistic genetic association between a polymorphic locus and a disease has been claimed to yield an odds ratio between 1.1 and 1.5 [17]. Thus at least 1000 subjects should be required to detect this association, depending on the prevalence of polymorphism. However, studies typically report sample sizes from 100 to 300 and rarely above 1000 subjects $[16,18]$, justifying the use of metaanalysis to increase power.

The aim of this study is to attempt to answer whether there is an association between the 5-HTTLPR and PD. As the s allele of 5-HTTLPR is significantly involved in anxiety traits, the assumption is that this polymorphism should be involved in PD.

\section{Methods \\ The search}

- Electronic databases: studies were identified through PubMed (which encompasses Medline, Premedline, and HelthSTAR), PsychINFO, Lilacs and ISI. The PubMed search was run using the Mesh terms: ("Serotonin Plasma Membrane Transport Proteins" [MeSH] OR "5-HTTLPR" OR "5-HTT" OR "SLC6A4") AND "Panic Disorder" [MeSH]. In PsychINFO, Lilacs and ISI the following words were used: "Panic" AND ("serotonin transporter" OR "serotonergic transporter" OR "5-HTT" OR "5-HTTLPR" OR "SLC6A4"). This search strategy was run in June and rerun in April 2007, and included only human studies, with no language restrictions, and a time scope from January 1996 to April 2007, i.e. since the 5-HTTLPR was described by Heils [9] in 1996.

- Reference cross-checking: the list of references of included studies was searched looking for additional studies.

Contact with authors: efforts were made to contact all research groups of studies included in the analysis to identify unpublished data. Three authors replied and no additional study was identified.

\section{Inclusion/Exclusion criteria}

Studies reporting the 5-HTTLPR in PD patients of any ethnic origin were evaluated by the authors. The inclusion criteria for this systematic review were: (1) type of studies: case-control and family-based studies; (2) type of participants: diagnoses of PD according to standard diagnostic criteria (DSM or ICD); (3) Hardy-Weinberg Equilibrium (HWE). Testing for HWE is commonly used for quality control of large-scale genotyping and is one of the few ways to identify systematic genotyping errors in unrelated individuals [19], and its assumption is required for the allele analysis [20].

Exclusion criteria included (1) studies in which the main disorders were other than PD; (2) replicated data (a part of sample used for more than one publication); (3) insufficient data to perform statistical analysis (unable even after contacting authors). Regarding replicated data, the included study was selected based on the sample size and the availability of information studied in this meta-analysis. Family studies were not included in the analyses because there is only one study published [21], so no analysis could be performed.

This meta-analysis methodology was performed according to MOOSE (Meta-analysis of Observational Studies in Epidemiology) group guidelines [22] and stages of this methodology are presented in a flowchart here in (Figure 1). 


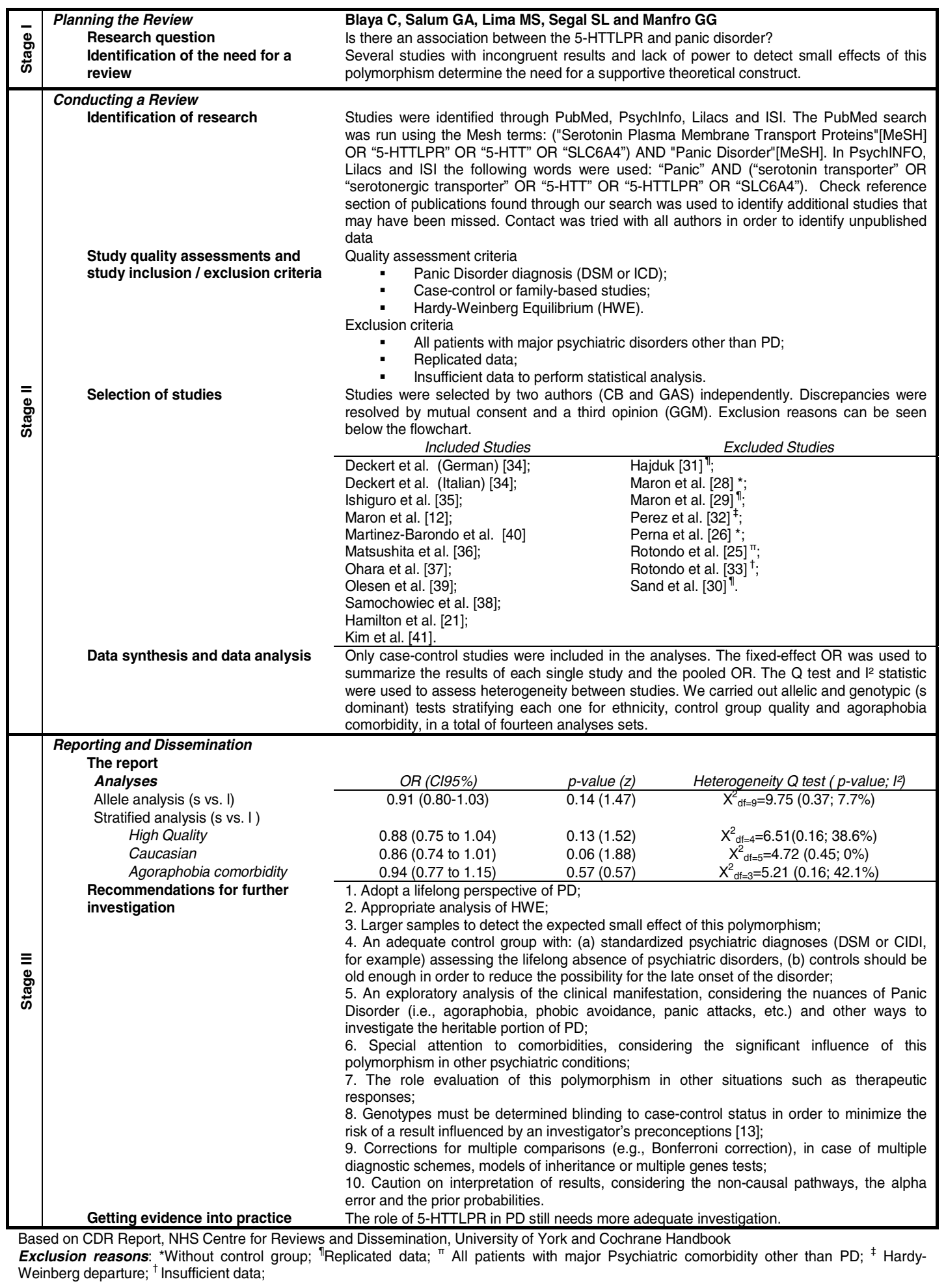

\section{Figure I}

Flowchart - Stages of Systematic Review with Meta-analysis. 


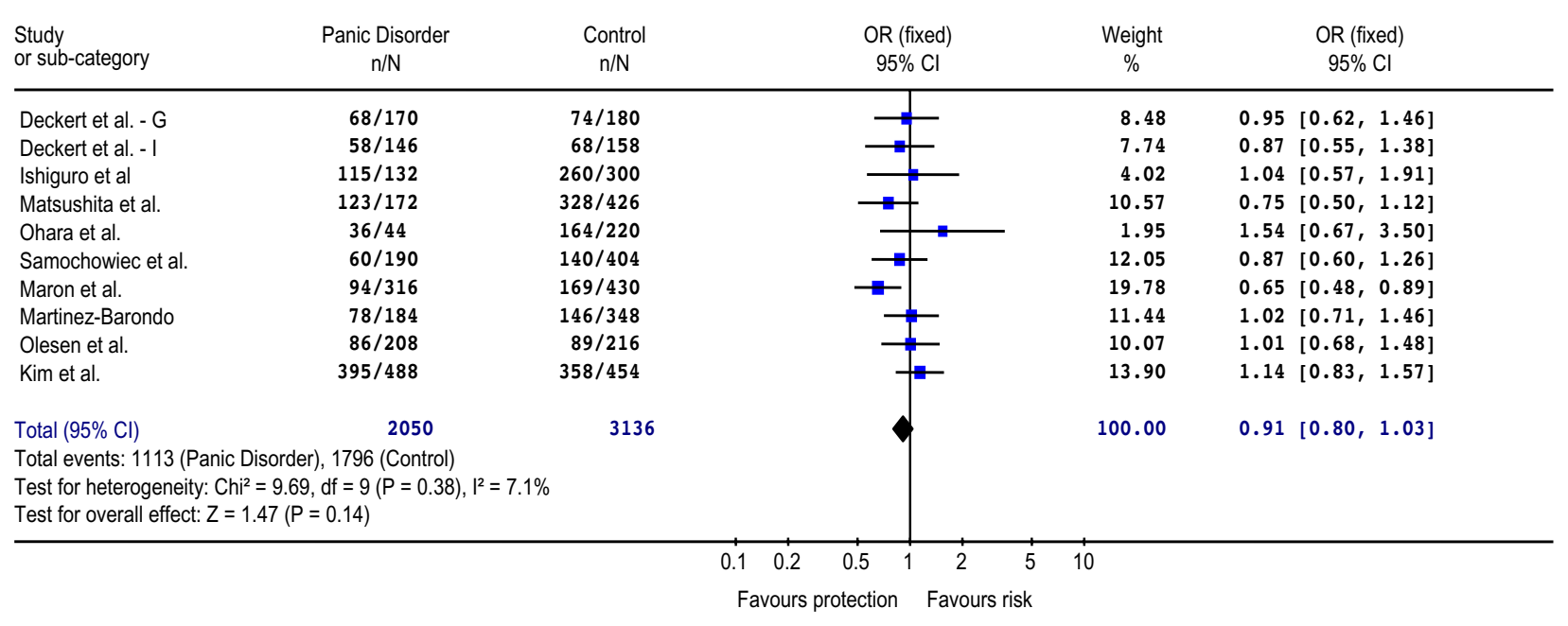

Figure 2

Forest plot with odds ratio of each study and pooled odds ratio for the association between the s allele and risk of Panic Disorder.

\section{Data extraction}

Data was independently extracted by two investigators (CB and GAS) using a standardized data extraction form. Discrepancies were resolved by discussion and if consensus was not achieved the decision was made by another reviewer (GGM). Data extracted included number of PD patients and controls for each of the three genotype groups (ll, ls, ss) in each eligible study. Only data regarding 5-HTTLPR and panic disorder were extracted regardless of other polymorphisms, disorders or outcomes reported in the studies. The male/female ratio, mean age, predominant ethnicity of the sample and psychiatric comorbidity were also extracted. Genotype frequencies were used to calculate whether they deviate significantly from HWE. In cases where data was not available in the published reports, authors were contacted directly. The information was classified as not reported when authors did not return to at least three contact trials or did not have this data available.

\section{Statistical analysis}

First, an analysis by alleles was performed because this analysis increases the power to detect differences and is the most used analysis in the current literature. Secondly, we analyze the most used model of inheritance (s dominant) to express directly the ll genotype risk [16]. In order to investigate interaction or confusion between studies, a stratified analysis was run after separating studies by ethnicity, quality of control groups and comorbidity with Agoraphobia, as recommended by Munafo and Flint [15].
It was considered as high quality control groups those with appropriate assessment diagnoses. As we performed fourteen different analyses sets of the same genetic data we also present correct p-values according to Finner's modification of the Bonferroni's procedure for multiple comparisons. Despite being an important analysis, we could not perform a stratified analysis for comorbidity with mood disorders and another anxiety disorders because this data was poorly described in studies included in this meta-analysis. We choose to perform an exploratory analysis because the heritable portion of PD that could be related to this polymorphism is not yet defined.

Asymptotic DerSimonian and Laird's Q test was used to assess heterogeneity. Because tests of heterogeneity may be underpowered to detect heterogeneity between studies when the number of studies is small [23], we also explore heterogeneity quantitatively using the $\mathrm{I}^{2}$ statistic [24].

The results of individual studies, as well as the pooled odds ratio (OR), were synthesized by the fixed effect model with $95 \%$ confidence intervals. The significance of the pooled OR was determined by the $\mathrm{z}$ test. Publication bias was assessed with a funnel plot (allele analyses). The analyses were done with Review Manager 4.2.8, software developed by the Cochrane Collaboration. The Power Calculator for Genetic Studies software was used in order to estimate the minimum OR that could be detected in this meta-analysis. 


\section{Results}

In total, 19 potential publications were identified, and 10 studies were included in this meta-analysis. No analysis was performed with family-based studies because only one was found in our search [21]. One study was excluded because all patients had Bipolar Disorder [25], three studies were excluded because there were no control groups [26-28], three studies were excluded because data were replicated [29-31], one study was excluded due to significant deviation of Hardy-Weinberg equilibrium [32] and one study [33] was excluded due to insufficient data. As one study [34] had two different sub samples clinically assessed with different methodologies, it was considered as two separate studies (Deckert German and Deckert Italian), resulting in ten studies included in our analysis (figure 1). The funnel plot suggests a couple of missing studies with OR higher than 1, but the small number of included trials does not allow drawing conclusions about publication bias.

Across all 10 case-control studies investigating 5-HTTLPR and panic disorder [12,34-41], a total of 1,025 patients and 1,568 controls were included, totaling 2,050 alleles among patients and 3,136 alleles between controls. Table 1 depicts data extracted from the included studies. Regarding the allele and stratified analyses, no evidence of heterogeneity was found. The statistics of heterogeneity $Q$ test and $\mathrm{I}^{2}$ are shown in the figure 2 . We found very similar results between the OR calculated by the random-effects model and Peto OR.

In the first analysis, including 5,186 alleles, we compared the 5-HTTLPR allelic distribution between patients with PD and controls of all ten studies included. The pooled $\mathrm{OR}=0.91$ was not significant (CI95\% 0.80 to $1.03 ; \mathrm{z}=$ $1.47 ; \mathrm{p}=0.14)$. The results of this analysis are shown in the forest plot (figure 2). Regarding family studies, we only identified one in this search [21]. The study had evaluated 340 individuals in 45 families with at least three affected people. No linkage between the 5-HTTLPR and PD was observed ( $\mathrm{p}=0.40)$.

As the included studies have subjects with different ethnicity and control groups with different quality, we performed stratified analyses. Only five studies - Maron et al. [12], Deckert Italian [34], Samochowiec et al. [38], Olesen et al. [39] and Kim et al. [41] - were classified as having high quality controls. Results showed no significant association between the s allele and PD in both analyses (high and low quality). Regarding the ethnic stratified analysis, no significant association was found in Caucasian or Asian separated analyses. Some studies evaluated the comorbidity with Agoraphobia in their exploratory analysis $[12,35,38,41]$ and no significant association was found in this stratified analysis. Table 2 depicts all these stratified analyses for the allelic analysis and genotype analysis.

We estimated the minimum OR that could be detected in this meta-analysis by allelic analysis. Considering a power of $90 \%$, a PD prevalence of $1.8 \%$ in Caucasoid [42], a mean frequency of s allele of 0.45 in a joint analysis with the Power Calculator for genetic studies, the minimum OR that we are capable to found is 1.14 .

\section{Discussion}

This is the first systematic review between the 5-HTTLPR and PD and no statistically significant association was found. In the overall analysis, there was no evidence for heterogeneity among the studies. This indicates no greater variation among the studies than could be expected by chance and provides the validity to the meta-analysis by suggesting that studies included in this analysis are comparable. However, no association was found between this polymorphism and PD even in all stratified analyses.

The main problem in genetic studies and psychiatric disorders is probably the lack of a phenotype definition. Clinical diagnoses according to DSM-IV may be heterogeneous constructs that combine elements with distinct genetic influences [43]. Additionally, psychiatric disorders usually overlap, and comorbidity might be a bias that impairs some associations. Therefore, some authors are interested in defining "intermediate" phenotypes that might have more direct expression of genes influencing a complex disorder and may have a simpler genetic architecture [43].

Also, a recently detected genetic variation (16A allele) [44] in the long allele of 5-HTTLPR has been linked to a lower expression of the serotonin transporter $[45,46]$. No study has evaluated this polymorphism neither in panic disorder nor in anxiety traits. Hence, studies should combine the evaluation of different polymorphisms that influence the protein expression.

\section{Limitations of this study}

The publication bias could not be adequately assessed in our meta-analysis by the funnel plot due to the small number of studies available. However, we believe that our search strategy was comprehensive enough. Moreover, additional sources of trials like contacting authors of included trials yield no additional study.

The sample size found in our meta-analysis is below that which is required to identify associations with minor effects [17]. In the sub-group analysis the sample size became even smaller. Thus, further investigation using larger sample sizes, in which the control group is properly diagnosed and ethnicity is evaluated, is needed. Such 
Table I: Included Case-control Studies of 5-HTTLPR and Panic Disorder

\begin{tabular}{|c|c|c|c|c|c|c|c|c|c|c|c|c|c|c|}
\hline \multirow[b]{2}{*}{$\begin{array}{l}\text { Studie } \\
\mathbf{S}\end{array}$} & \multirow[b]{2}{*}{$\begin{array}{c}\text { Group } \\
\text { s (n) }\end{array}$} & \multirow[b]{2}{*}{$\begin{array}{c}\text { Male } \\
(\%)\end{array}$} & \multirow[b]{2}{*}{$\begin{array}{c}\text { Diagn } \\
\text { osis }\end{array}$} & \multirow[b]{2}{*}{$\begin{array}{c}\text { Como } \\
\text { rbidit } \\
y\end{array}$} & \multirow[b]{2}{*}{$\begin{array}{c}\text { Mean } \\
\text { Age }\end{array}$} & \multirow[b]{2}{*}{ Origin } & \multicolumn{3}{|c|}{$\begin{array}{c}\text { Genotype Frequencyl } \\
\text { Total (\%) }\end{array}$} & \multicolumn{2}{|c|}{$\begin{array}{c}\text { Alelle } \\
\text { frequencyl } \\
\text { Total (\%) }\end{array}$} & \multicolumn{3}{|c|}{ HWE reported $(\mathrm{df}=\mathrm{I})$} \\
\hline & & & & & & & ss & sl & II & s & $\mathbf{I}$ & & $\mathbf{X}^{2}$ & $\mathbf{p}$ \\
\hline \multirow[t]{2}{*}{$\begin{array}{c}\text { Ishigur } \\
\text { o et al. } \\
\text { [35] }\end{array}$} & $\begin{array}{l}\text { Panic } \\
(66)\end{array}$ & $\begin{array}{c}30 \\
(45.5)\end{array}$ & $\begin{array}{l}\text { DSM- } \\
\text { IV }\end{array}$ & NR & 40 & $\begin{array}{c}\text { Japanes } \\
\mathrm{e}\end{array}$ & $\begin{array}{l}51 / 66 \\
(77.3)\end{array}$ & $\begin{array}{l}13 / 66 \\
(19.7)\end{array}$ & $\begin{array}{c}2 / 66 \\
(3)\end{array}$ & $\begin{array}{l}115 / \\
132 \\
(87.1)\end{array}$ & $\begin{array}{l}17 / 132 \\
(12.9)\end{array}$ & YES & 0.986 & 0.321 \\
\hline & $\begin{array}{l}\text { Control } \\
\text { (150) }\end{array}$ & $\begin{array}{c}70 \\
(46.7)\end{array}$ & $\begin{array}{l}\text { Not } \\
\text { report } \\
\text { ed }\end{array}$ & & 41 & & $\begin{array}{l}114 / \\
150 \\
(76)\end{array}$ & $\begin{array}{c}32 / 150 \\
(21.3)\end{array}$ & $\begin{array}{c}4 / 150 \\
(2.7)\end{array}$ & $\begin{array}{c}260 / \\
300 \\
(86.7)\end{array}$ & $\begin{array}{c}40 / 300 \\
(13.3)\end{array}$ & YES & 0.887 & 0.346 \\
\hline \multirow[t]{2}{*}{$\begin{array}{l}\text { Matsus } \\
\text { hita et } \\
\text { al. [36] }\end{array}$} & $\begin{array}{l}\text { Panic } \\
(86)\end{array}$ & $\begin{array}{c}54 \\
(62.7)\end{array}$ & $\begin{array}{l}\text { DSM- } \\
\text { III-R }\end{array}$ & NR & 37.0 & $\begin{array}{c}\text { Japanes } \\
\mathrm{e}\end{array}$ & $\begin{array}{l}44 / 86 \\
(51.2)\end{array}$ & $\begin{array}{l}35 / 86 \\
(40.7)\end{array}$ & $\begin{array}{l}7 / 86 \\
(8.1)\end{array}$ & $\begin{array}{c}123 / \\
172 \\
(71.5)\end{array}$ & $\begin{array}{c}49 / 172 \\
(28.5)\end{array}$ & YES & $<0.001$ & 0.992 \\
\hline & $\begin{array}{l}\text { Control } \\
(2 / 3)\end{array}$ & $\begin{array}{c}96 \\
(45.1)\end{array}$ & $\begin{array}{l}\text { Not } \\
\text { report } \\
\text { ed }\end{array}$ & & 37.7 & & $\begin{array}{c}125 / \\
213 \\
(58.7)\end{array}$ & $\begin{array}{c}78 / 213 \\
(36.6)\end{array}$ & $\begin{array}{c}10 / 213 \\
(4.7)\end{array}$ & $\begin{array}{l}328 / \\
426 \\
(77)\end{array}$ & $\begin{array}{c}98 / 426 \\
(23)\end{array}$ & YES & 0.242 & 0.623 \\
\hline \multirow[t]{2}{*}{$\begin{array}{l}\text { Decker } \\
\text { t et al. } \\
\text { Germa } \\
\text { n [34] }\end{array}$} & $\begin{array}{l}\text { Panic } \\
\text { (85) }\end{array}$ & NR & $\begin{array}{l}\text { DSM- } \\
\text { III-R } \\
\text { and } \\
\text { CIDI }\end{array}$ & $\begin{array}{c}\text { Yes } \\
\text { (Mood) } \\
; \\
\text { Anxiet } \\
\text { y NR }\end{array}$ & NR & $\underset{n}{\text { Germa }}$ & $\begin{array}{l}12 / 66 \\
(14.1)\end{array}$ & $\begin{array}{l}44 / 66 \\
(51.8)\end{array}$ & $\begin{array}{l}29 / 66 \\
(34.1)\end{array}$ & $\begin{array}{c}68 / 170 \\
(40)\end{array}$ & $\begin{array}{l}102 / \\
170 \\
(60)\end{array}$ & NO & 0.523 & 0.470 \\
\hline & $\begin{array}{c}\text { Control } \\
(90)\end{array}$ & NR & None & & NR & & $\begin{array}{l}16 / 90 \\
(17.8)\end{array}$ & $\begin{array}{l}42 / 90 \\
(46.7)\end{array}$ & $\begin{array}{l}32 / 90 \\
(35.6)\end{array}$ & $\begin{array}{c}74 / 180 \\
(41.1)\end{array}$ & $\begin{array}{c}106 / \\
180 \\
(58.9)\end{array}$ & YES & 0.118 & 0.731 \\
\hline \multirow[t]{2}{*}{$\begin{array}{c}\text { Decker } \\
\text { t et al. } \\
\text { Italian } \\
{[34]}\end{array}$} & $\begin{array}{l}\text { Panic } \\
\text { (73) }\end{array}$ & NR & $\begin{array}{l}\text { DSM- } \\
\text { III-R } \\
\text { and } \\
\text { SADS- } \\
\text { LA }\end{array}$ & $\begin{array}{c}\text { Mood } \\
\text { absent; } \\
\text { Anxiet } \\
\text { y NR }\end{array}$ & NR & Italian & $\begin{array}{l}13 / 73 \\
(17.8)\end{array}$ & $\begin{array}{l}32 / 73 \\
(43.8)\end{array}$ & $\begin{array}{l}28 / 73 \\
(38.4)\end{array}$ & $\begin{array}{c}58 / 146 \\
(39.7)\end{array}$ & $\begin{array}{c}88 / 146 \\
(60.3)\end{array}$ & NO & 0.523 & 0.470 \\
\hline & $\begin{array}{c}\text { Control } \\
\text { (79) }\end{array}$ & NR & $\begin{array}{l}\text { DSM- } \\
\text { III-R } \\
\text { and } \\
\text { DISt }\end{array}$ & & NR & & $\begin{array}{l}12 / 79 \\
(15.2)\end{array}$ & $\begin{array}{l}44 / 79 \\
(55.7)\end{array}$ & $\begin{array}{l}23 / 79 \\
(29.1)\end{array}$ & $\begin{array}{c}68 / 158 \\
(43)\end{array}$ & $\begin{array}{c}90 / 158 \\
(57)\end{array}$ & YES & 1.460 & 0.227 \\
\hline \multirow[t]{2}{*}{$\begin{array}{l}\text { Ohara } \\
\text { et al. } \\
\text { [37] }\end{array}$} & $\begin{array}{l}\text { Panic } \\
(22)\end{array}$ & II (50) & $\begin{array}{l}\text { DSM- } \\
\text { IV }\end{array}$ & $\begin{array}{c}\text { Yes } \\
\text { (Anxiet } \\
\text { y) }\end{array}$ & 35.1 & $\begin{array}{c}\text { Japanes } \\
\mathrm{e}\end{array}$ & $\begin{array}{l}14 / 22 \\
(63.6)\end{array}$ & $\begin{array}{c}8 / 22 \\
(36.4)\end{array}$ & $\begin{array}{c}0 / 22 \\
(0)\end{array}$ & $\begin{array}{l}36 / 44 \\
(81.8)\end{array}$ & $\begin{array}{c}8 / 44 \\
(18.2)\end{array}$ & NO & 1.086 & 0.769 \\
\hline & $\begin{array}{l}\text { Control } \\
\text { (II0) }\end{array}$ & NR & $\begin{array}{l}\text { Not } \\
\text { report } \\
\text { ed }\end{array}$ & & NR & & $\begin{array}{l}62 / 110 \\
(56.4)\end{array}$ & $\begin{array}{c}40 / 110 \\
(36.4)\end{array}$ & $\begin{array}{c}8 / 110 \\
(7.3)\end{array}$ & $\begin{array}{l}164 / \\
220 \\
(74.5)\end{array}$ & $\begin{array}{c}56 / 220 \\
(25.5)\end{array}$ & NO & 0.192 & 0.661 \\
\hline \multirow[t]{2}{*}{$\begin{array}{c}\text { Samoc } \\
\text { howiec } \\
\text { et al. } \\
{[38]}\end{array}$} & $\begin{array}{l}\text { Panic } \\
\text { (95) }\end{array}$ & $\begin{array}{c}24 \\
(23.8)\end{array}$ & CIDI & $\begin{array}{c}\text { Yes } \\
\text { (Anxiet } \\
y)\end{array}$ & 38.7 & $\begin{array}{c}\text { Polonai } \\
\text { se }\end{array}$ & $\begin{array}{l}10 / 95 \\
(10.5)\end{array}$ & $\begin{array}{l}40 / 95 \\
(42.1)\end{array}$ & $\begin{array}{l}45 / 95 \\
(47.4)\end{array}$ & $\begin{array}{c}60 / 190 \\
(31.6)\end{array}$ & $\begin{array}{c}130 / \\
190 \\
(68.4)\end{array}$ & NO & 0.062 & 0.803 \\
\hline & $\begin{array}{l}\text { Control } \\
\text { (202) }\end{array}$ & $\begin{array}{c}54 \\
(26.7)\end{array}$ & ICD-10 & & 35.9 & & $\begin{array}{c}22 / 202 \\
(10.9)\end{array}$ & $\begin{array}{c}96 / 202 \\
(47.5)\end{array}$ & $\begin{array}{c}84 / 202 \\
(41.6)\end{array}$ & $\begin{array}{c}140 / \\
404 \\
(34.7)\end{array}$ & $\begin{array}{c}264 / \\
404 \\
(65.3)\end{array}$ & NO & 0.492 & 0.483 \\
\hline \multirow[t]{2}{*}{$\begin{array}{c}\text { Maron } \\
\text { et al. } \\
\text { [12] }\end{array}$} & $\begin{array}{l}\text { Panic } \\
\text { (158) }\end{array}$ & $\begin{array}{c}32 \\
(20.2)\end{array}$ & $\begin{array}{l}\text { DSM- } \\
\text { IV } \\
\text { MINI }\end{array}$ & $\begin{array}{c}\text { Yes } \\
\text { (Anxiet } \\
y \text { and } \\
\text { Mood) }\end{array}$ & 38.0 & $\begin{array}{c}\text { Estonia } \\
\mathrm{n}\end{array}$ & $\begin{array}{l}11 / 158 \\
(7)\end{array}$ & $\begin{array}{c}72 / 158 \\
(45.6)\end{array}$ & $\begin{array}{c}75 / 158 \\
(47.5)\end{array}$ & $\begin{array}{c}94 / 316 \\
(29.7)\end{array}$ & $\begin{array}{c}222 / \\
316 \\
(70.3)\end{array}$ & YES & 1.288 & 0.256 \\
\hline & $\begin{array}{l}\text { Control } \\
(215)\end{array}$ & $56(26)$ & $\mathrm{MINI}+$ & & 39.8 & & $\begin{array}{c}34 / 215 \\
(15.8)\end{array}$ & $\begin{array}{l}101 / \\
215 \\
(47)\end{array}$ & $\begin{array}{c}80 / 215 \\
(37.2)\end{array}$ & $\begin{array}{c}169 / \\
430 \\
(39.3)\end{array}$ & $\begin{array}{c}261 / \\
430 \\
(60.7)\end{array}$ & YES & 0.051 & 0.822 \\
\hline \multirow[t]{2}{*}{$\begin{array}{l}\text { Olesen } \\
\text { et al. } \\
\text { [39] }\end{array}$} & $\begin{array}{l}\text { Panic } \\
(104)\end{array}$ & $\begin{array}{c}28 \\
(26.9)\end{array}$ & $\begin{array}{l}\text { DSM- } \\
\text { IV }\end{array}$ & $\begin{array}{c}\text { Yes } \\
\text { (Anxiet } \\
\text { y) }\end{array}$ & NR & Danish & $\begin{array}{l}15 / 104 \\
(14.4)\end{array}$ & $\begin{array}{c}56 / 104 \\
(53.8)\end{array}$ & $\begin{array}{c}33 / 104 \\
(31.7)\end{array}$ & $\begin{array}{c}86 / 208 \\
(41.3)\end{array}$ & $\begin{array}{l}122 / \\
208 \\
(58.7)\end{array}$ & NO & 1.263 & 0.261 \\
\hline & $\begin{array}{c}\text { Control } \\
(108)\end{array}$ & $\begin{array}{c}30 \\
(27.7)\end{array}$ & $\begin{array}{c}\text { Clinical } \\
\text { intervie } \\
\text { w }\end{array}$ & & NR & & $\begin{array}{l}18 / 108 \\
(16.7)\end{array}$ & $\begin{array}{c}53 / 108 \\
(49.1)\end{array}$ & $\begin{array}{c}37 / 108 \\
(34.3)\end{array}$ & $\begin{array}{c}89 / 216 \\
(41.2)\end{array}$ & $\begin{array}{l}127 \mid \\
216 \\
(58.8)\end{array}$ & NO & 0.018 & 0.894 \\
\hline
\end{tabular}


Table I: Included Case-control Studies of 5-HTTLPR and Panic Disorder (Continued)

\begin{tabular}{|c|c|c|c|c|c|c|c|c|c|c|c|c|c|c|}
\hline \multirow{2}{*}{$\begin{array}{c}\text { Martin } \\
\text { ez- } \\
\text { Barond } \\
\text { o et al. } \\
\text { [40] }\end{array}$} & $\begin{array}{l}\text { Panic } \\
\text { (92) }\end{array}$ & $\begin{array}{c}28 \\
(30.4)\end{array}$ & $\begin{array}{l}\text { DSM- } \\
\text { IV }\end{array}$ & NR & 35.8 & Spanish & $\begin{array}{l}18 / 92 \\
(19.6)\end{array}$ & $\begin{array}{l}42 / 92 \\
(45.7)\end{array}$ & $\begin{array}{l}32 / 92 \\
(34.8)\end{array}$ & $\begin{array}{l}78 / 184 \\
(42.4)\end{array}$ & $\begin{array}{c}106 / \\
184 \\
(57.6)\end{array}$ & $\mathrm{NO}$ & 0.392 & 0.531 \\
\hline & $\begin{array}{l}\text { Control } \\
(174)\end{array}$ & $\begin{array}{c}67 \\
(38.5)\end{array}$ & $\begin{array}{l}\text { Not } \\
\text { report } \\
\text { ed }\end{array}$ & & 38.4 & & $\begin{array}{c}36 / 174 \\
(20.7)\end{array}$ & $\begin{array}{c}74 / 174 \\
(42.5)\end{array}$ & $\begin{array}{c}64 / 174 \\
(36.8)\end{array}$ & $\begin{array}{l}146 / \\
348 \\
(42)\end{array}$ & $\begin{array}{l}202 / \\
348 \\
(58)\end{array}$ & NO & 2.798 & 0.094 \\
\hline \multirow[t]{2}{*}{$\begin{array}{l}\text { Kim et } \\
\text { al. [4I] }\end{array}$} & $\begin{array}{l}\text { Panic } \\
(244)\end{array}$ & $\begin{array}{c}143 \\
(58.6)\end{array}$ & $\begin{array}{l}\text { DSM- } \\
\text { IV }\end{array}$ & $\begin{array}{c}\text { Yes } \\
\text { (Mood) }\end{array}$ & 36.1 & Korean & $\begin{array}{c}159 / \\
244 \\
(65.2)\end{array}$ & $\begin{array}{c}77 / 244 \\
(31.6)\end{array}$ & $\begin{array}{c}8 / 244 \\
(3.3)\end{array}$ & $\begin{array}{c}395 / \\
488 \\
(80.9)\end{array}$ & $\begin{array}{c}93 / 488 \\
(19.1)\end{array}$ & YES & 0.1278 & 0.721 \\
\hline & $\begin{array}{l}\text { Control } \\
(227)\end{array}$ & $\begin{array}{c}102 \\
(44.9)\end{array}$ & $\begin{array}{c}\text { Clinical } \\
\text { Intervi } \\
\text { ew }\end{array}$ & & 33.1 & & $\begin{array}{c}141 / \\
227 \\
(62.1)\end{array}$ & $\begin{array}{c}76 / 227 \\
(33.5)\end{array}$ & $\begin{array}{c}10 / 227 \\
(4.4)\end{array}$ & $\begin{array}{c}358 / \\
454 \\
(78.9)\end{array}$ & $\begin{array}{c}96 / 454 \\
(21.1)\end{array}$ & YES & 0.0035 & 0.953 \\
\hline
\end{tabular}

Note: NR, Not reported; HWE, Hardy Weinberg Equilibrium; DSM-IV, Diagnostic and Statistical Manual of Mental Disorders IV; ICD-I0, International Classification of Diseases; CIDI, Composite International Diagnostic Interview; MINI, MINI International Neuropsychiatric Interview. tControls with first degree relatives without psychiatric disorders.

studies should focus on exploratory analyses in order to identify the heritable features that might be related to this polymorphism. Additionally, we could not perform a subgroup analysis divided by gender, because only one study reported this data. Neither did we find any stratified analyses regarding co-morbidity (with alcohol, mood and anxiety disorders), even though associations between the 5-HTTLPR and these psychiatric disorders have been reported $[30,47,48]$.

This meta-analysis was carried out with case-control studies. However, some authors [13] suggest that the results from this design finding a positive association between the genome and a disorder could be false. Additionally, a positive result from a case-control study might be due to population stratification rather than linkage disequilibrium [49] affected by the different disease prevalence and different marker frequencies in the subpopulation thereby producing spurious associations [50,51]. The use of Genomic Control, although with limitations [52], may be an alternative in analyzing data of case-control studies. Unfortunately, we could not perform an additional analysis with family studies because we identified only one study with this design. Family-based studies have a unique design in which population stratification is controlled.

\section{Conclusion}

In summary, results from this systematic review do not support the hypothesis of a significant association between 5-HTTLPR and PD. All the sub-analyses performed failed to find an association between PD and this polymorphism. However, more studies are needed in different ethnic populations in order to evaluate a possible minor effect. Finally, the 5-HTTLPR does not seem to play a major role in the genetics of panic disorder and, therefore, other polymorphisms should be investigated.

\section{Abbreviations}

PD, Panic Disorder

SSRIs, Serotonin Selective Reuptake Inhibitors

5-HTTLPR, Serotonin Transporter Promoter Polymorphism

DSM, Diagnostic and Statistical Manual

ICD, International Classification of Diseases

CIDI, Composite International Diagnostic Interview

MINI, MINI International Neuropsychiatric Interview.

5-HTT, Serotoninergic transporter gene

SLC6A4, Solute Carrier Family 6 (Neurotransmitter transporter serotonin), member 4

HWE, Hardy-Weinberg Equilibrium

MOOSE, Meta-analysis of Observational Studies in Epidemiology

OR, Odds ratio

CI95\%, Confident Interval of 95\%

\section{Competing interests}

Mauricio S. Lima is medical director of Eli Lilly do Brazil.

Carolina Blaya and Gisele G. Manfro are speakers of Eli Lilly Brazil.

Giovanni A. Salum and Sandra Leistner-Segal have no competing interests. 
Table 2: Results of the overall studies and sub-group studies stratified by ethnicity, quality of control group and comorbidity with Agoraphobia

\begin{tabular}{|c|c|c|c|c|c|c|c|c|}
\hline & \multirow[t]{2}{*}{$n^{\mathrm{a}}$} & \multirow[t]{2}{*}{ OR $(95 \% \mathrm{Cl})$} & \multicolumn{3}{|c|}{ Significance } & \multicolumn{3}{|c|}{ Heterogeneity } \\
\hline & & & $\mathrm{Z}$ & $\mathrm{p}$-value & Finner's $p$ & $\chi^{2 c}$ & $p$-value & $\left.\right|^{2}$ \\
\hline $\begin{array}{l}\text { Allelic } \\
\text { analyses (s/ } \\
\text { I) }\end{array}$ & 10 & $\begin{array}{l}0.91(0.80- \\
1.03)\end{array}$ & 1.47 & 0.14 & 0.58 & 9.75 & 0.37 & $7.7 \%$ \\
\hline \multicolumn{9}{|l|}{ Ethnicity } \\
\hline Caucasians & 6 & $\begin{array}{l}0.86(0.74- \\
1.01)\end{array}$ & 1.88 & 0.06 & 0.58 & 4.72 & 0.45 & $0 \%$ \\
\hline Asians & 4 & $\begin{array}{l}1.02(0.81- \\
1.27)\end{array}$ & 0.14 & 0.89 & 0.92 & 3.67 & 0.30 & $18.3 \%$ \\
\hline \multicolumn{9}{|l|}{$\begin{array}{l}\text { Quality of } \\
\text { Control } \\
\text { Group }\end{array}$} \\
\hline $\begin{array}{l}\text { High } \\
\text { quality } \\
\text { control } \\
\text { group }\end{array}$ & 5 & $\begin{array}{l}0.88(0.75- \\
1.04)\end{array}$ & 1.52 & 0.13 & 0.58 & 6.51 & 0.16 & $38.6 \%$ \\
\hline $\begin{array}{l}\text { Low } \\
\text { quality } \\
\text { control } \\
\text { group }\end{array}$ & 5 & $\begin{array}{l}0.96(0.78- \\
1.17)\end{array}$ & 0.43 & 0.67 & 0.76 & 2.87 & 0.58 & $0 \%$ \\
\hline \multicolumn{9}{|l|}{$\begin{array}{l}\text { Agoraphobi } \\
\text { a } \\
\text { comorbidity }\end{array}$} \\
\hline $\begin{array}{l}\text { With } \\
\text { Agoraphob } \\
\text { ia }\end{array}$ & 4 & $\begin{array}{l}0.94(0.77- \\
1.15)\end{array}$ & 0.57 & 0.57 & 0.69 & 5.21 & 0.16 & $42.4 \%$ \\
\hline $\begin{array}{l}\text { Without } \\
\text { Agoraphob } \\
\text { ia }\end{array}$ & 3 & $\begin{array}{l}0.80(0.60- \\
1.08)\end{array}$ & 1.48 & 0.14 & 0.58 & 2.30 & 0.32 & $12.9 \%$ \\
\hline $\begin{array}{l}\text { Genotype } \\
\text { (Is+ss) vs. II }\end{array}$ & 10 & $\begin{array}{l}0.87(0.71- \\
1.06)\end{array}$ & 1.39 & 0.17 & 0.58 & 7 & 0.64 & $0 \%$ \\
\hline \multicolumn{9}{|l|}{ Ethnicity } \\
\hline Caucasians & 6 & $\begin{array}{l}0.85(0.68- \\
1.05)\end{array}$ & 1.48 & 0.14 & 0.58 & 4.36 & 0.50 & $0 \%$ \\
\hline Asians & 4 & $\begin{array}{l}1.01(0.55- \\
1.86)\end{array}$ & 0.03 & 0.98 & 0.98 & 2.56 & 0.46 & $0 \%$ \\
\hline \multicolumn{9}{|l|}{$\begin{array}{l}\text { Quality of } \\
\text { Control } \\
\text { Group }\end{array}$} \\
\hline $\begin{array}{l}\text { High } \\
\text { quality } \\
\text { control } \\
\text { group }\end{array}$ & 5 & $\begin{array}{l}0.80(0.63- \\
1.03)\end{array}$ & 1.76 & 0.08 & 0.58 & 3.72 & 0.45 & $0 \%$ \\
\hline $\begin{array}{l}\text { Low } \\
\text { quality } \\
\text { control } \\
\text { group }\end{array}$ & 5 & $\begin{array}{l}1.02(0.71- \\
1.46)\end{array}$ & 0.11 & 0.91 & 0.93 & 2.30 & 0.68 & $0 \%$ \\
\hline \multicolumn{9}{|l|}{$\begin{array}{l}\text { Agoraphobi } \\
\text { a } \\
\text { comorbidity }\end{array}$} \\
\hline $\begin{array}{l}\text { With } \\
\text { Agoraphob } \\
\text { ia }\end{array}$ & 4 & $\begin{array}{l}0.80(0.57- \\
\mathrm{I} .1 \mathrm{I})\end{array}$ & 1.32 & 0.19 & 0.58 & 2.90 & 0.41 & $0 \%$ \\
\hline $\begin{array}{l}\text { Without } \\
\text { Agoraphob } \\
\text { ia }\end{array}$ & 3 & $\begin{array}{l}0.85(0.51- \\
1.40)\end{array}$ & 0.65 & 0.51 & 0.67 & 1.18 & 0.55 & $0 \%$ \\
\hline
\end{tabular}

Abbreviations: OR, odds ratio (fixed effect); $Q$, Cochran's $x^{2}$-based $Q$ statistic test used to assess the heterogeneity; $z$ test used to determine the significance of the overall OR; $I^{2}$, inconsistency; Finner's p-value, adjusted p-value for multiple comparison

a The number of studies included are indicated

$\mathrm{b}$ The first allele is the risk allele

c Degrees of freedom $=$ number of studies $-\mathrm{I}$. 


\section{Authors' contributions}

CB conceived of the study, extracted the data, participated in the design and drafted the manuscript. GAS extracted the data, participated in the design, drafted the manuscript and performed the statistical analysis. MSL helped in the statistical analysis and helped to draft the manuscript. SLS helped to draft the manuscript. GGM, participated in its design, coordination and drafted the manuscript. All authors read and approved the final manuscript.

\section{Acknowledgements}

This study was supported by Fundação de Amparo à Pesquisa from Hospital de Clínicas de Porto Alegre (FIPE-HCPA 04-272) (CB and GGM). The funding was used for publication processing charge.

\section{References}

I. Crowe RR, Noyes R, Pauls DL, Slymen D: A family study of panic disorder. Arch Gen Psychiatry 1983, 40:1065-1069.

2. Inada Y, Yoneda H, Koh J, Sakai J, Himei A, Kinoshita Y, Akabame K, Hiraoka Y, Sakai T: Positive association between panic disorder and polymorphism of the serotonin $2 A$ receptor gene. Psychiatry Res 2003, I | 8:25-3I.

3. Kendler KS, Neale MC, Kessler RC, Heath AC, Eaves L]: Panic disorder in women: a population-based twin study. Psychol Med 1993, 23:397-406.

4. Hettema JM, Neale MC, Kendler KS: A review and meta-analysis of the genetic epidemiology of anxiety disorders. Am J Psychiatry 2001, 158:1568-1578

5. Deckert J, Catalano M, Syagailo YV, Bosi M, Okladnova O, Di Bella D, Nothen MM, Maffei P, Franke P, Fritze J, Maier W, Propping P, Beckmann $\mathrm{H}$, Bellodi L, Lesch KP: Excess of high activity monoamine oxidase $A$ gene promoter alleles in female patients with panic disorder. Hum Mol Genet 1999, 8:621-624.

6. Versiani M, Cassano G, Perugi G, Benedetti A, Mastalli L, Nardi A, Savino M: Reboxetine, a selective norepinephrine reuptake inhibitor, is an effective and well-tolerated treatment for panic disorder. J Clin Psychiatry 2002, 63:3 I-37.

7. Lesch KP, Balling U, Gross J, Strauss K, Wolozin BL, Murphy DL, Riederer $\mathrm{P}$ : Organization of the human serotonin transporter gene. J Neural Transm Gen Sect 1994, 95: I57-162.

8. Lesch KP, Bengel D, Heils A, Sabol SZ, Greenberg BD, Petri S, Benjamin J, Muller CR, Hamer DH, Murphy DL: Association of anxiety-related traits with a polymorphism in the serotonin transporter gene regulatory region. Science 1996, 274: $|527-| 53 \mid$.

9. Heils A, Teufel A, Petri S, Stober G, Riederer P, Bengel D, Lesch KP Allelic variation of human serotonin transporter gene expression. J Neurochem 1996, 66:2621-2624.

10. Brown JS, Joiner TE: The serotonin transporter gene's association with mental disorder: a meta analysis. In Department of Psychology Miami, The Florida State University; 2003:33.

II. Sen S, Burmeister M, Ghosh D: Meta-analysis of the association between a serotonin transporter promoter polymorphism (5-HTTLPR) and anxiety-related personality traits. Am J Med Genet 2004, I 27B:85-89.

12. Maron E, Lang A, Tasa G, Liivlaid L, Toru I, Must A, Vasar V, Shlik J: Associations between serotonin-related gene polymorphisms and panic disorder. Int I Neuropsychopharmacol 2005, 8:26I-266

13. Sullivan PF, Eaves LJ, Kendler KS, Neale MC: Genetic case-control association studies in neuropsychiatry. Arch Gen Psychiatry 2001 58:1015-1024

14. Hirschhorn JN, Lohmueller K, Byrne E, Hirschhorn K: A comprehensive review of genetic association studies. Genet Med 2002, 4:45-6I.

15. Munafo MR, Flint J: Meta-analysis of genetic association studies. Trends Genet 2004, 20:439-444.

16. Salanti G, Amountza G, Ntzani EE, loannidis JP: Hardy-Weinberg equilibrium in genetic association studies: an empirical eval- uation of reporting, deviations, and power. Eur J Hum Genet 2005, 13:840-848.

17. loannidis JP: Genetic associations: false or true? Trends Mol Med 2003, 9:135-138.

18. Ioannidis JP: Molecular bias. Eur J Epidemiol 2005, 20:739-745.

19. Wittke-Thompson JK, Pluzhnikov A, Cox NJ: Rational inferences about departures from Hardy-Weinberg equilibrium. Am J Hum Genet 2005, 76:967-986.

20. Sasieni PD: From genotypes to genes: doubling the sample size. Biometrics 1997, 53:1253-|26I.

21. Hamilton SP, Heiman GA, Haghighi F, Mick S, Klein DF, Hodge SE, Weissman MM, Fyer AJ, Knowles JA: Lack of genetic linkage or association between a functional serotonin transporter polymorphism and panic disorder. Psychiatr Genet 1999, 9: I-6.

22. Stroup DF, Berlin JA, Morton SC, Olkin I, Williamson GD, Rennie D, Moher D, Becker BJ, Sipe TA, Thacker SB: Meta-analysis of observational studies in epidemiology: a proposal for reporting. Meta-analysis Of Observational Studies in Epidemiology (MOOSE) group. Jama 2000, 283:2008-20I2.

23. Hardy RJ, Thompson SG: Detecting and describing heterogeneity in meta-analysis. Stat Med 1998, I7:84I-856.

24. Takkouche B, Cadarso-Suarez C, Spiegelman D: Evaluation of old and new tests of heterogeneity in epidemiologic meta-analysis. Am J Epidemiol 1999, I50:206-215.

25. Rotondo A, Mazzanti C, Dell'Osso L, Rucci P, Sullivan P, Bouanani S, Gonnelli C, Goldman D, Cassano GB: Catechol o-methyltransferase, serotonin transporter, and tryptophan hydroxylase gene polymorphisms in bipolar disorder patients with and without comorbid panic disorder. Am J Psychiatry 2002, 159:23-29.

26. Perna G, Favaron E, Di Bela D, Bussi R, Bellodi L: Antipanic efficacy of paroxetine and polymorphism within the Promoter of the Serotonin Transporter Gene. Neuropsychopharmacology 2005.

27. Perna G, di Bella D, Favaron E, Cucchi M, Liperi L, Bellodi L: Lack of relationship between $\mathrm{CO} 2$ reactivity and serotonin transporter gene regulatory region polymorphism in panic disorder. Am J Med Genet B Neuropsychiatr Genet 2004, I 29:4I-43.

28. Maron E, Tasa G, Toru I, Lang A, Vasar V, Shlik J: Association between serotonin-related genetic polymorphisms and CCK-4-induced panic attacks with or without 5-hydroxytryptophan pretreatment in healthy volunteers. World J Biol Psychiatry 2004, 5: I 49-I54.

29. Maron E, Nikopensius T, Koks S, Altmae S, Heinaste E, Vabrit K, Tammekivi V, Hallast P, Koido K, Kurg A, Metspalu A, Vasar E, Vasar V, Shlik J: Association study of $\mathbf{9 0}$ candidate gene polymorphisms in panic disorder. Psychiatr Genet 2005, 15:17-24.

30. Sand P, Lesch KP, Catalano M, Bosi M, Syagailo YV, Okladnova O, Di Bella D, Maffei P, Heils A, Friess F, Politi E, Nothen MM, Franke P, Stober G, Fritze J, Maier W, Propping P, Beckmann H, Bellodi L, Riederer P, Deckert J: Polymorphic MAO-A and 5-HT-transporter genes: analysis of interactions in panic disorder. World J Biol Psychiatry 2000, I:147-150.

31. Hajduk A: [A search for psychobiological determinants of anxiety disorders]. Ann Acad Med Stetin 2004, 50:65-75.

32. Perez M, Brown JS, Vrshek-Schallhorn S, Johnson F, Joiner TE Jr.: Differentiation of obsessive-compulsive-, panic-, obsessivecompulsive personality-, and non-disordered individuals by variation in the promoter region of the serotonin transporter gene. J Anxiety Disord 2005.

33. Rotondo A, Mazzanti C, Michelini S, Gemignani A, Lezza A, Capovani B, Bouanani S, Coli E, Miniati M, Pfanner C, Presta S, Milanfranchi A Ramacciotti C, Dell'Osso L, Mauri M, Cassano G, Goldman D: Association study of panic, obsessive-compulsive and eating disorders with a functional polymorphism of the serotonin transporter promoter. European Neuropsychopharmachology 1997, 7:236.

34. Deckert I Catalano M, Heils A, Di Bella D, Friess F, Politi E, Franke $P$, Nothen MM, Maier W, Bellodi L, Lesch KP: Functional promoter polymorphism of the human serotonin transporter: lack of association with panic disorder. Psychiatr Genet 1997, 7:45-47.

35. Ishiguro H, Arinami T, Yamada K, Otsuka Y, Toru M, Shibuya H: An association study between a transcriptional polymorphism in the serotonin transporter gene and panic disorder in a Japanese population. Psychiatry Clin Neurosci 1997, 5 I:333-335. 
36. Matsushita S, Muramatsu T, Kimura M, Shirakawa O, Mita T, Nakai T, Higuchi S: Serotonin transporter gene regulatory region polymorphism and panic disorder. Mol Psychiatry 1997, 2:390-392.

37. Ohara K, Nagai M, Suzuki Y, Ochiai M: Association between anxiety disorders and a functional polymorphism in the serotonin transporter gene. Psychiatry Res 1998, 81:277-279.

38. Samochowiec J, Hajduk A, Samochowiec A, Horodnicki J, Stepien G, Grzywacz A, Kucharska-Mazur J: Association studies of MAO-A, COMT, and 5-HTT genes polymorphisms in patients with anxiety disorders of the phobic spectrum. Psychiatry Res 2004, I 28:2|-26.

39. Olesen OF, Bennike B, Hansen ES, Koefoed P, Woldbye DP, Bolwig TG, Mellerup E: The short/long polymorphism in the serotonin transporter gene promoter is not associated with panic disorder in a Scandinavian sample. Psychiatr Genet 2005, 15:159.

40. Martinez-Barrondo S, Saiz PA, Morales B, Garcia-Portilla MP, Coto E, Álvarez $\mathrm{V}$, Bobes J: Serotonin gene polymorphisms in patients with panic disorder. Acta Esp Psiquiatr 2005, 33:210-215.

41. Kim W, Choi YC, Yoon KS, Cho DY, Pae CU, Woo JM: Tryptophan hydroxylase and serotonin transporter gene polymorphism does not affect the diagnosis, clinical features and treatment outcome of panic disorder in the Korean population. Prog Neuropsychopharmacol Biol Psychiatry 2006, 30:14|3-1418.

42. Goodwin RD, Faravelli C, Rosi S, Cosci F, Truglia E, de Graaf R, Wittchen HU: The epidemiology of panic disorder and agoraphobia in Europe. Eur Neuropsychopharmacol 2005, I5:435-443.

43. Smoller JW, Rosenbaum JF, Biederman J, Kennedy J, Dai D, Racette SR, Laird NM, Kagan J, Snidman N, Hirshfeld-Becker D, Tsuang MT, Sklar PB, Slaugenhaupt SA: Association of a genetic marker at the corticotropin-releasing hormone locus with behavioral inhibition. Biol Psychiatry 2003, 54:1376-I38I.

44. Ozaki N, Goldman D, Kaye WH, Plotnicov K, Greenberg BD, Lappalainen J, Rudnick G, Murphy DL: Serotonin transporter missense mutation associated with a complex neuropsychiatric phenotype. Molecular Psychiatry 2003, 8:933-936.

45. Kilic F, Murphy DL, Rudnick G: A human serotonin transporter mutation causes constitutive activation of transport activity. Molecular Pharmacology 2003, 64:440-446.

46. Hu XZ, Lipsky RH, Zhu G, Akhtar LA, Taubman J, Greenberg BD, Xu K, Arnold PD, Richter MA, Kennedy JL, Murphy DL, Goldman D: Serotonin transporter promoter gain-of-function genotypes are linked to obsessive-compulsive disorder. Am J Hum Genet 2006, 78:8I5-826.

47. Feinn R, Nellissery M, Kranzler HR: Meta-analysis of the association of a functional serotonin transporter promoter polymorphism with alcohol dependence. Am J Med Genet B Neuropsychiatr Genet 2005, 133:79-84.

48. Lasky-Su JA, Faraone SV, Glatt SJ, Tsuang MT: Meta-analysis of the association between two polymorphisms in the serotonin transporter gene and affective disorders. Am J Med Genet B Neuropsychiatr Genet 2005, I33: I I0-I I5.

49. Li CC: Population subdivision with respect to multiple alleles. Ann Hum Genet 1969, 33:23-29.

50. Shmulewitz D, Zhang J, Greenberg DA: Case-control association studies in mixed populations: correcting using genomic control. Hum Hered 2004, 58: 145-153.

5I. Bacanu SA, Devlin B, Roeder K: The power of genomic control. Am J Hum Genet 2000, 66:1 1933-1944.

52. Devlin B, Roeder K, Wasserman L: Genomic control, a new approach to genetic-based association studies. Theor Popul Biol 200I, 60:155-166 htp://ww.behavioralandbrainfunctions.com/content/3/1/41 